\section{Consumo alimentar e dislipidemia decorrente da terapia antirretroviral combinada para infecção pelo HIV: uma revisão sistemática}

\author{
Dietary intake and dyslipidemia arising from combination \\ antiretroviral therapy for HIV infection: a systematic review
}

Luara Bellinghausen Almeida ${ }^{1}$, Kelly Virecoulon Giudici², Patricia Constante Jaime ${ }^{3}$

\begin{abstract}
RESUMO
Revisar e sintetizar as evidências científicas disponíveis sobre a relação entre o consumo alimentar e dislipidemia em pacientes infectados pelo HIV em terapia antirretroviral combinada de alta atividade (TARV). Desenvolveu-se uma revisão sistemática de literatura. Foram pesquisados estudos originais e duas categorias de exposição dietética foram revisadas: consumo de energia e nutriente ou consumo de uma dieta teste. Foi feita síntese narrativa dos estudos selecionados. Os achados foram sintetizados segundo a categoria de desfecho metabólico (efeito sobre colesterol total e LDL-c, efeito sobre HDL-c e efeito sobre triglicérides). Vinte estudos originais foram incluídos na revisão, sendo 13 ensaios clínicos e 7 estudos epidemiológicos observacionais. A suplementação com ácido graxo $\omega-3$ resultou em significativa redução nos níveis séricos de triglicérides. Observou-se evidência insuficiente acerca da efetividade de intervenções dietéticas na prevenção e controle das dislipidemias em pacientes infectados pelo HIV em uso de TARV. Arq Bras Endocrinol Metab. 2009;53(5):519-27.
\end{abstract}

Palavras-chave

AIDS; HIV; síndrome lipodistrófica do HIV; dislipidemias; dieta; consumo alimentar

\begin{abstract}
To review and synthesize the available scientific evidence on the relationship between dietary intake and dyslipidemias in HIV-infected patients in combination antiretroviral therapy (ART). A systematic review of literature was carried out. Original and published studies were investigated and two categories of dietary exposure were considered: energy and nutrient intake, and consumption of a test diet. A narrative review of included studies was conducted. The findings were summarized according to category of metabolic outcomes (effect on total cholesterol and LDL-c, effect on HDL-c and effect on triglycerides). Twenty original studies were included in this review, being 13 clinical trials and 7 observational studies. $\omega-3$ fatty acid supplementation led to a significant decrease in triglycerides. There was very little evidence on the effectiveness of dietary interventions for the prevention and control of dyslipidemias in HIV-infected patients receiving ART. Arq Bras Endocrinol Metab. 2009;53(5):519-27.
\end{abstract}
Trabalho desenvolvido no Departamento de Nutrição da FSP/USP
1 Faculdade de Saúde Pública da Universidade de São Paulo (FSP/USP), São Paulo, SP, Brasil ${ }^{2}$ Departamento de Nutrição da FSP/USP, São Paulo, SP, Brasil ${ }^{3}$ Departamento de Nutrição; Programa de Pós-Graduação Nutrição em Saúde Pública da FSP/USP, São Paulo, SP, Brasil

Correspondência para: Patricia Constante Jaime Departamento de Nutrição, Faculdade de Saúde Pública da Universidade de São Paulo Av. Doutor Arnaldo, 715, 2 andar 01246-904 - São Paulo, SP, Brasil constant@usp.br

Recebido em 7/Mar/2009 Aceito em 26/Abr/2009

Key words

AIDS; HIV; lipodystrophy syndrome HIV; dyslipidemias; diet; food consumption

\section{INTRODUÇÃO}

A terapia antirretroviral combinada de alta atividade - (TARV) causou um profundo impacto na história da infecção pelo HIV com drástica diminuição da mortalidade e da ocorrência de infecções oportunistas e subsequente aumento da sobrevida dos portadores (1). No
Brasil, a queda da mortalidade por AIDS evidencia o atual caráter crônico e o controle da evolução da doença (2).

Por outro lado, eventos adversos associados à terapêutica vêm sendo descritos em pessoas com HIV em uso de TARV, trazendo outras demandas aos serviços responsáveis pelo cuidado integral a esses pacientes. 
Nesse cenário, destaca-se a síndrome lipodistrófica do HIV, caracterizada por dislipidemias, alterações glicêmicas e alterações morfológicas com lipoatrofia e lipohipertrofia $(3,4)$.

A dislipidemia associada à infecção pelo HIV caracteriza-se por baixos níveis séricos de HDL colesterol (HDL-c) e elevação de colesterol total (CT), LDL colesterol (LDL-c) e triglicérides (TGC), constituindo perfil sabidamente aterogênico (4). Alguns estudos observacionais revelaram que a incidência de evento cardiovascular em pacientes infectados pelo HIV em TARV é maior do que aquela observada na população em geral (5-7).

Uma imbricada e, ainda, não conclusiva rede de causalidade explica a ocorrência de tais alterações lipídicas, envolvendo a infecção em si pelo HIV (8), o tipo de droga utilizada e o esquema terapêutico antirretroviral (7-9) e fatores de risco tradicionais, como dieta aterogênica $(8,10,11)$ e inatividade física $(12,13)$.

Na população geral, um extenso corpo de evidências comprova a relação entre o consumo alimentar, alterações de perfil lipídico e ocorrência de doenças cardiovasculares $(14,15)$. A intervenção dietoterápica tem sido recomendada para a prevenção e controle primário da hipercolesterolemia e da hipertrigliceridemia nos pacientes infectados pelo HIV em uso de TARV $(8,16)$.

O objetivo do presente estudo foi sistematizar e avaliar as evidências científicas disponíveis sobre a relação entre o consumo alimentar e dislipidemia em pacientes infectados pelo HIV em uso de TARV, visando a informar melhor as condutas de aconselhamento nutricional para prevenção e controle de dislipidemias nesta população.

\section{METODOLOGIA}

Desenvolveu-se um estudo de revisão sistemática de literatura. A pergunta que direcionou a revisão foi: "Qual é a relação entre a dieta (consumo alimentar) e dislipidemias em indivíduos infectados pelos HIV e em uso de TARV?”

\section{Critérios de inclusão e exclusão de estudos}

Foram pesquisados estudos originais que avaliaram a relação entre consumo alimentar e hipercolesterolemia (valores aumentados de LDL-c ou CT), hipertrigliceridemia (valores aumentados de triglicérides) e valores reduzidos de HDL-c em indivíduos infectados pelo HIV em uso de TARV. Duas categorias de exposição dietética foram consideradas: consumo de energia e nu- triente ou consumo de uma dieta teste. Em relação ao delineamento do estudo, foram aceitos os estudos epidemiológicos observacionais e os ensaios clínicos (aleatorizados ou não, controlados ou não).

\section{Estratégia de busca e seleção de estudos}

A estratégia de busca dos estudos originais foi desenhada para ser usada no PubMed e adaptada a outras duas bases de dados (SciELO e Lilacs). Consideraramse os seguintes limites de busca: estudos em humanos, publicados em inglês, português, espanhol, francês ou italiano e data limite de publicação em janeiro de 2009. Três conjuntos de intersecção de termos de busca bibliográfica foram combinados: população (HIV, AIDS, HAART ou antiretroviral therapy), exposição dietética (diet, food consumption, dietary intake, eating, food intake, energy intake, nutrient, nutritional support) $\mathrm{e}$ desfecho (lipodystrophy, metabolic syndrome HIV, dyslipidemias, hypercholesterolemia, triglycerides, lipid metabolism).

A triagem e a extração dos dados dos artigos foram feitas por dois dos autores (LBA e KVG). Os artigos foram rejeitados na primeira triagem nos casos de os revisores determinarem, a partir da análise do título e do resumo, inadequação aos critérios de inclusão ou presença de algum dos critérios de exclusão da revisão. Nos casos de incerteza ou discordância, o texto completo do artigo era consultado para confirmar sua elegibilidade. Em complementação à busca nas bases de dados bibliográficas, as listas de referências bibliográficas dos artigos incluídos foram consultadas para identificar algum possível estudo relevante não identificado anteriormente.

\section{Síntese e comparação dos estudos}

Os artigos foram sistematicamente revisados em relação ao uso de TARV (esquema de combinação de drogas antirretrovirais, uso de inibidor de protease, tempo de uso), delineamento do estudo (observacional ou ensaio clínico), tipo de intervenção feita nos casos de ensaios clínicos, presença de cointervenção (fármacos hipolipemiantes, atividade física e controle do tabagismo), método de avaliação de consumo alimentar (recordatório alimentar de 24 horas, questionário de frequência alimentar, diário alimentar ou outro), variáveis dietéticas e metabólicas exploradas e, finalmente, tipo de efeito ou associação testado e encontrado. Nos casos dos estudos que envolveram indivíduos infectados pelo HIV em uso ou não de TARV, confirmou-se elegibilidade somente 
se foi possível avaliar separadamente, nos pacientes em TARV, o efeito da dieta sobre níveis séricos de triglicérides, HDL-c, CT e/ou LDL-c.

Foi feita síntese narrativa dos estudos selecionados. $\mathrm{O}$ uso da metanálise foi inapropriado devido à marcada heterogeneidade no delineamento dos estudos, nas intervenções testadas, nas variáveis dietéticas investigadas e nas estratégias de avaliação de consumo alimentar. Os achados foram então sintetizados em tabelas segundo categoria de desfecho metabólico (efeito sobre CT e LDL-c, efeito sobre HDL-c e efeito sobre triglicérides séricos).

\section{RESULTADOS}

A estratégia de pesquisa encontrou 365 artigos não duplicados, tendo sido a grande maioria localizada por meio da primeira consulta feita ao PubMed (360 artigos). Ao final, 20 artigos de estudos originais foram incluídos na revisão. O processo completo de seleção dos estudos está descrito na figura 1 .

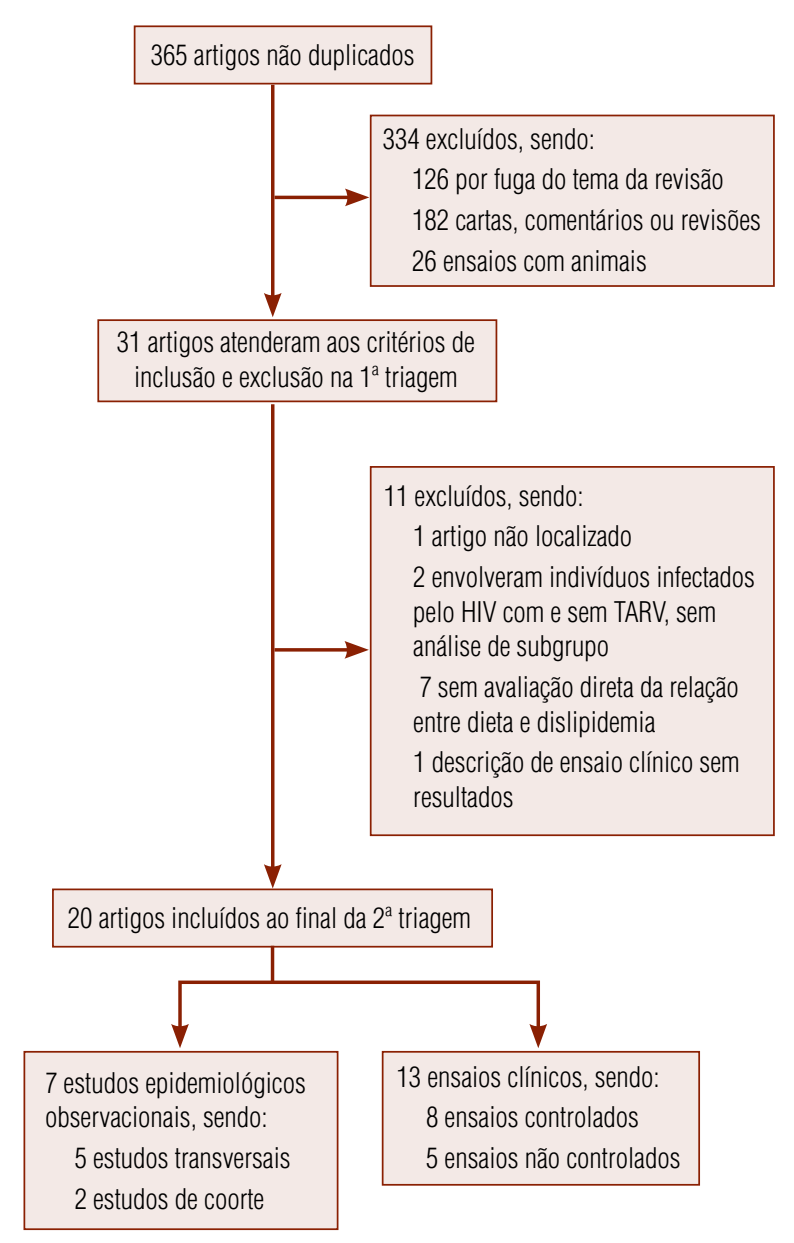

Figura 1. Seleção dos estudos.
Os estudos foram desenvolvidos em países ao redor do mundo, sendo a maioria norte-americanos (17-24), três australianos (25-27), dois brasileiros $(28,29)$, seis europeus (30-35) e um canadense (36). Treze estudos experimentais avaliaram o efeito de dietas sobre as dislipidemias, abrangendo intervenções distintas: apenas dieta $(\mathrm{n}=1)$, dieta e exercício físico estruturado $(\mathrm{n}=$ 1), dieta e fármacos hipolipemiantes $(\mathrm{n}=5)$, dieta $\mathrm{e}$ suplementação com ácido graxo poli-insaturado $\omega-3$ (n = 5), dieta e administração de ácido graxo tetradeciltioacético $(n=1)$. Entre os estudos observacionais, cinco transversais e dois longitudinais investigaram associações entre nutrientes da dieta e níveis de lipídeos séricos. As características sobre delineamento, população e desfechos estão contempladas na tabela 1 .

Sobre as dietas testadas nos ensaios clínicos, genericamente definidas como dieta para redução de colesterol e triglicérides, elas são descritas como dieta hipolipídica, hipolipídica rica em fibras e ainda a dieta preconizada pela American Heart Association (reduzida em gorduras saturadas e trans, rica em frutas, vegetais e grãos integrais, produtos lácteos com baixo teor de gorduras, peixes e carnes magras), em muitos casos associando-se orientação nutricional e recomendações para mudanças no estilo de vida.

\section{Efeito sobre CT e LDL-c}

O desfecho colesterol total foi avaliado em 19 estudos e o LDL-c, em 11, como mostrado na tabela 2, com resultados mais consistentes para colesterol total.

Entre os experimentais incluídos nesta revisão, o estudo de Barrios e cols. (33) foi o único que avaliou o efeito isolado da intervenção dietética sobre dislipidemia relacionada à TARV, indicando redução média de $11 \%$ da concentração sérica de colesterol total após 3 meses para pacientes com boa adesão à dieta hipolipídica. Os demais estudos aplicam a intervenção dietética associada a cointervenções ou como padrão de controle para outros tipos de intervenção em teste (exercício físico, drogas hipolipemiantes, suplementação com ácido graxo).

Melroe e cols. (17) e Lima e cols. (28) desenvolveram estudos não controlados baseados nas recomendações do National Cholesterol Education Program (37) para verificar a efetividade na redução lipídica e na prevenção cardiovascular para pacientes em TARV. Tais recomendações baseiam-se em modificações no estilo de vida como abordagem primária e adição de tratamento farmacológico em caso de persistência das dislipidemias associadas ao risco cardiovascular. Reduções sig- 
nificativas para colesterol total e LDL-c são observadas, indicando que as intervenções multicomponentes são bem-sucedidas, no entanto, sem a possibilidade de se destacar o efeito isolado da dieta. Nos ensaios clínicos controlados incluídos nesta revisão e que compararam dietoterapia e dietoterapia somada à terapia medicamentosa, efeitos expressivos dos fármacos e menores da dieta no tratamento da hipercolesterolemia foram apresentados $(25,26,35)$.

$\mathrm{O}$ efeito da dieta associada à suplementação com ácido graxo $\omega-3$ foi verificado em quatro estudos, com poucas evidências de benefícios para a redução de colesterol (18,30-32). Somente na pesquisa realizada por De Truchis e cols. (32) os resultados mostraram redução nos valores de colesterol total $(-0,4 \%)$ para o grupo que recebeu dieta e suplemento de $\omega-3$. Essa pequena variação contribui para efeito significativo em comparação ao grupo controle, que recebeu orientação dietética e placebo $(\neq-8,5 \%, \mathrm{p}=0,0117)$, portanto, representando um achado isolado e em discordância com os resultados dos demais estudos, que apontam ausência de efeito hipocolesterolêmico dos ácidos graxos $\omega$-3.

Os estudos observacionais sinalizam associações entre os níveis séricos de lipídeos e alguns nutrientes da dieta. O colesterol total relacionou-se positivamente com o percentual de proteínas totais e de origem animal (2l) e com o teor de lipídeos totais da dieta (22). Para LDL-c, associação positiva foi detectada com consumo de álcool e associação inversa com consumo de fibras.

\section{Efeito sobre HDL-c}

O desfecho HDL-c foi investigado em 14 estudos (Tabela 3 ). Em três intervenções com fármacos hipolipemiantes, apenas uma encontrou variação significativa, porém no sentido indesejado de redução significativa do HDL-c, em comparação ao grupo que só recebeu intervenção dietética $(\mathrm{p}=0,01)(26)$. Não se encontrou efeito associado à suplementação com $\omega$-3 ou à exposição ao exercício físico estruturado sobre os níveis séricos de HDL $(18,29,30,32)$. Já nos estudos transversais, de Hadigan e cols. (19) e Flooris-Moore e cols. (22), observou-se, respectivamente, associação positiva com o consumo de álcool e fibras dietéticas e os níveis séricos de HDL.

\section{Efeito sobre triglicérides séricos}

Entre os desfechos estudados, as intervenções dietéticas e farmacológicas contemplam resultados de maior con- sistência e magnitude sobre os triglicérides séricos, sobretudo em relação aos efeitos da suplementação com ácidos graxos $\omega$-3.

Reduções de $12 \%$ e $23 \%$ nos triglicérides foram observadas, respectivamente após 3 e 6 meses da prescrição de dieta hipolipídica, sendo o nível de adesão à dieta um fator determinante para os resultados favoráveis (33).

As intervenções multicomponentes sobre estilo de vida associadas à utilização de fármacos hipolipemiantes resultam em significativas reduções nos níveis séricos de triglicérides, com potencial efetividade no controle da hipertrigliceridemia secundária à TARV $(17,28)$.

Todos os ensaios clínicos que avaliaram a suplementação com ácido graxo $\omega$-3 indicam positivo impacto na redução dos níveis de triglicérides séricos, evidenciando outra importante abordagem terapêutica no manejo da hipertrigliceridemia $(18,27,30$ 32). Um estudo italiano (31) comparou a redução nos níveis de triglicérides para 156 indivíduos acompanhados por 18 meses e divididos em três grupos: a) controle (dieta e exercício físico), b) intervenção com terapia farmacológica (fibratos) e $c$ ) intervenção com suplemento de $\omega$-3 (1 grama/dia). A porcentagem de normalização dos níveis de triglicérides foi de $8,2 \%$ no Grupo A contra 34\% no Grupo B e $25,9 \%$ no Grupo C, sendo que o grupo controle difere significativamente dos outros dois de intervenção $(\mathrm{p}<0,001)$ mas não há diferença estatística entre os grupos B e C. Taxas significativas de redução nos triglicérides, variando de $25 \%$ a $57 \%$, foram observadas nos grupos com suplementação de $\omega$ - 3 nos demais estudos avaliados, com baixa ocorrência de relatos de efeitos colaterais entre os pacientes, apontando uma alternativa de tratamento a fim de evitar a toxicidade e interação medicamentosa proveniente do uso de grande quantidade de fármacos em indivíduos vivendo com AIDS.

Sobre a administração do ácido graxo tetradeciltioacético associado à dieta hipolipídica, foram observados efeitos potencializadores na redução dos triglicérides e também do colesterol total em estudo norueguês conduzido por Fredrisken e cols. (34).

Correlações positivas foram encontradas entre os níveis de triglicérides e fatores dietéticos, como consumo de proteínas totais e proteína animal, gordura total, gordura saturada e gordura trans $(21,24)$. 


\begin{tabular}{|c|c|c|c|c|c|}
\hline Estudo & $\begin{array}{l}\text { Delineamento/ } \\
\text { Seguimento }\end{array}$ & $\begin{array}{l}\text { População } \\
\text { Intervenção/ } \\
\text { Total, Sexo } \\
\end{array}$ & Exposição & $\begin{array}{l}\text { Desfechos } \\
\text { metabólicos }\end{array}$ & Estatística \\
\hline \multicolumn{6}{|l|}{ Experimentais } \\
\hline Barrios e cols. (33) & $\begin{array}{l}\text { Ensaio clínico } \\
\text { não controlado } \\
12 \text { semanas }\end{array}$ & 230 / 230, M e F & Dieta hipolipídica & $\mathrm{TG}, \mathrm{CT}$ & $\begin{array}{l}\text { Testes t-Student e } \\
\text { Mann-Whitney }\end{array}$ \\
\hline Terry e cols. (29) & $\begin{array}{l}\text { Ensaio clínico controlado } \\
12 \text { semanas }\end{array}$ & 15/30, M e F & $\begin{array}{l}\text { GC: Dieta (não especificada) } \\
\text { Gl: Dieta (não especificada) + exercício físico estruturado }\end{array}$ & TG, CT e HDL & $\begin{array}{l}\text { ANOVA para medidas } \\
\text { repetidas }\end{array}$ \\
\hline Melroe e cols. (17) & $\begin{array}{l}\text { Ensaio clínico } \\
\text { não controlado } \\
20 \text { meses }\end{array}$ & 26/26, M e F & $\begin{array}{l}\text { Recomendações do NCEP: } \\
\text { Dieta American Heart Association + orientações para } \\
\text { estilo de vida + fármaco hipolipemiante (gemfibrozil e/ } \\
\text { ou artovastatina) }\end{array}$ & TG, CT & Teste t-Student \\
\hline Lima e cols. (28) & $\begin{array}{l}\text { Ensaio clínico } \\
\text { não controlado } \\
24 \text { semanas }\end{array}$ & $87 / 87, \mathrm{M} \mathrm{e} \mathrm{F}$ & $\begin{array}{l}\text { Dieta da American Heart Association + orientações } \\
\text { para estilo de vida + fármacos hipolipemiantes } \\
\text { (fibratos e estatinas) }\end{array}$ & $\begin{array}{l}\text { TG, CT, LDL e } \\
\text { HDL }\end{array}$ & $\begin{array}{l}\text { ANOVA para medidas } \\
\text { repetidas }\end{array}$ \\
\hline Moyle e cols. (35) & $\begin{array}{l}\text { Ensaio clínico controlado } \\
24 \text { semanas }\end{array}$ & 14/26, M & $\begin{array}{l}\text { GC: Dieta hipolipídica + orientações para estilo de vida } \\
\text { Gl: Dieta hipolipídica + orientações para estilo de vida } \\
\text { + fármaco hipolipemiante (pravastatina) }\end{array}$ & $\begin{array}{l}\text { TG, CT, LDL, } \\
\quad \text { HDL }\end{array}$ & $\begin{array}{l}\text { Teste t-Student e } \\
\text { Mann-Witney }\end{array}$ \\
\hline Miller e cols. (25) & $\begin{array}{l}\text { Ensaio clínico controlado } \\
16 \text { semanas }\end{array}$ & 17/37, M & $\begin{array}{l}\text { GC: Dieta (não especificada) + placebo } \\
\text { Gl: Dieta (não especificada) + fármaco hipolipemiante } \\
\text { (gemfibrozil) }\end{array}$ & TG, CT, HDL & ANOVA \\
\hline Mallon e cols. (26) & $\begin{array}{l}\text { Ensaio clínico controlado } \\
16 \text { semanas }\end{array}$ & $16 / 33, \mathrm{M}$ & $\begin{array}{l}\text { GC: Dieta (não especificada) + placebo } \\
\text { Gl: Dieta (não especificada) + fármaco hipolipemiante } \\
\text { (pravastatina) }\end{array}$ & $\mathrm{TG}, \mathrm{CT}, \mathrm{HDL}$ & $\begin{array}{l}\text { Teste Mann-Whitney. } \\
\text { AUC }\end{array}$ \\
\hline Ranieri (30) & $\begin{array}{l}\text { Ensaio clínico } \\
\text { não controlado } \\
24 \text { semanas }\end{array}$ & 20, M e F & $\begin{array}{l}\text { Dieta hipolipídica rica em fibras + orientações para } \\
\text { estilo de vida + suplemento } \omega-3\end{array}$ & TG, CT e LDL & Teste t-Student \\
\hline Wohl e cols. (18) & $\begin{array}{l}\text { Ensaio clínico controlado } \\
16 \text { semanas }\end{array}$ & 26/52, M e F & $\begin{array}{l}\text { GC: Dieta American Heart Association + orientação } \\
\text { para atividade física } \\
\text { Gl: Dieta American Heart Association + orientação para } \\
\text { atividade física + suplemento } \omega-3\end{array}$ & $\begin{array}{l}\text { TG, CT, LDLe } \\
\text { HDL }\end{array}$ & $\begin{array}{l}\text { Teste t-Student } \\
\text { pareado Teste de } \\
\text { Wilcoxon }\end{array}$ \\
\hline $\begin{array}{l}\text { Manfredi e cols. } \\
\text { (31) }\end{array}$ & $\begin{array}{l}\text { Ensaio clínico controlado } \\
18 \text { meses }\end{array}$ & $54 / 156, \mathrm{M} \mathrm{e} \mathrm{F}$ & $\begin{array}{l}\text { GC: Dieta (não especificada) e exercício físico } \\
\text { Gl-1: Tratamento farmacológico (fibratos) } \\
\text { Gl-2: Suplemento } \omega-3\end{array}$ & $\mathrm{TG}, \mathrm{CT}$ & $\mathrm{NI}$ \\
\hline Carter e cols. (27) & Ensaio clínico controlado & $5 / 11, \mathrm{M} \mathrm{e} \mathrm{F}$ & $\begin{array}{l}\text { GC: Dieta (não especificada) + placebo } \\
\text { Gl: Dieta (não especificada) + suplemento } \omega-3\end{array}$ & TG & Qui-quadrado \\
\hline $\begin{array}{l}\text { De Truchis e cols. } \\
\text { (32) }\end{array}$ & $\begin{array}{l}\text { Ensaio clínico controlado } \\
12 \text { semanas }\end{array}$ & $58 / 120, \mathrm{M} \mathrm{e} \mathrm{F}$ & $\begin{array}{l}\text { GC: Dieta American Heart Association + placebo } \\
\text { Gl: Dieta American Heart Association + suplemento } \\
\omega-3\end{array}$ & TG, CT e HDL & $\begin{array}{l}\text { Regressão linear e } \\
\text { covariância não } \\
\text { paramétrica }\end{array}$ \\
\hline $\begin{array}{l}\text { Fredriksen e cols. } \\
\text { (34) }\end{array}$ & $\begin{array}{l}\text { Ensaio clínico } \\
\text { não controlado } \\
8 \text { semanas }\end{array}$ & 10/10, M & $\begin{array}{l}\text { Semana 0-4: Dieta hipolipídica } \\
\text { Semana 4-8: Dieta hipolipídica + tetradeciltioacético* }\end{array}$ & $\begin{array}{l}\text { TG, CT, LDL e } \\
\text { HDL }\end{array}$ & $\begin{array}{l}\text { Teste de Wilcoxon } \\
\text { Correlação de } \\
\text { Spearman }\end{array}$ \\
\hline \multicolumn{6}{|l|}{ Observacionais } \\
\hline Hadigan e cols. (19) & Transversal & $85, \mathrm{MeF}$ & $\begin{array}{l}\text { Macronutrientes, álcool, fibra dietética, ácidos graxos } \\
\text { poli-insaturados e saturados, colesterol }\end{array}$ & $\begin{array}{c}\text { TG, CT, LDL e } \\
\text { HDL }\end{array}$ & $\begin{array}{l}\text { Regressão } \\
\text { multivariada }\end{array}$ \\
\hline Gavrila e cols. (20) & Transversal & $120, \mathrm{M} \mathrm{e} \mathrm{F}$ & $\begin{array}{l}\text { Macronutrientes, ácidos graxos saturados e } \\
\text { insaturados, colesterol, fibras, folato (dieta + } \\
\text { suplemento), vitamina E (dieta/suplemento) }\end{array}$ & $\begin{array}{l}\text { TG, CT, LDL e } \\
\text { HDL }\end{array}$ & $\begin{array}{l}\text { Regressão linear } \\
\text { bivariada, Testes } \\
\text { Mann-Whitney e } \\
\text { Kruskall-Wallis }\end{array}$ \\
\hline Shah e cols. (21) & Transversal & $45, M$ & $\begin{array}{l}\text { Macronutrientes, proteína animal, ácidos graxos trans, } \\
\text { fibras dietéticas }\end{array}$ & TG, CT e HDL & $\begin{array}{l}\text { Correlação de } \\
\text { Spearman Teste de } \\
\text { Wilcoxon }\end{array}$ \\
\hline $\begin{array}{l}\text { Floris-Moore e } \\
\text { cols. (22) }\end{array}$ & Transversal & $102, F$ & Lipídeos totais e fibras & $\begin{array}{c}\text { TG, CT, LDL e } \\
\text { HDL }\end{array}$ & $\begin{array}{l}\text { Análise univariada, } \\
\text { Correlação de } \\
\text { Spearman }\end{array}$ \\
\hline
\end{tabular}


Tabela 1. Estudos incluídos: descrição do delineamento, população, tipo de exposição, desfechos metabólicos e estatística (continuação)

\begin{tabular}{|c|c|c|c|c|c|}
\hline Estudo & $\begin{array}{l}\text { Delineamento/ } \\
\text { Seguimento }\end{array}$ & $\begin{array}{l}\text { População } \\
\text { Intervenção/ } \\
\text { Total, Sexo }\end{array}$ & Exposição & $\begin{array}{l}\text { Desfechos } \\
\text { metabólicos }\end{array}$ & Estatística \\
\hline \multicolumn{6}{|l|}{ Observacionais } \\
\hline $\begin{array}{l}\text { Aghdassi e cols. } \\
(36)\end{array}$ & Transversal & $75, \mathrm{MeF}$ & Medidas de cromo (dieta, plasma, unhas, urina) & $\begin{array}{l}\text { TG, CT, LDL e } \\
\text { HDL }\end{array}$ & $\begin{array}{l}\text { Correlação de } \\
\text { Spearman }\end{array}$ \\
\hline Wanke e cols. (23) & $\begin{array}{l}\text { Coorte } \\
4 \text { anos }\end{array}$ & $49, \mathrm{MeF}$ & $\begin{array}{l}\text { Calorias, macronutrientes, ácidos graxos saturados e } \\
\text { insaturados, açúcar, fibras dietéticas, vitaminas A, C, E, } \\
\text { B1; selênio, zinco, ferro, folato }\end{array}$ & $\begin{array}{l}\text { TG, CT, LDL e } \\
\text { HDL }\end{array}$ & Correlação de Pearson \\
\hline Joy e cols. (24) & $\begin{array}{l}\text { Coorte } \\
7 \text { anos }\end{array}$ & $356, \mathrm{M} \mathrm{e} F$ & $\begin{array}{l}\text { Lipídeos, ácidos graxos saturados, ácidos graxos trans } \\
\text { e colesterol }\end{array}$ & TG, CT e LDL & $\begin{array}{l}\text { Modelo de regressão } \\
\text { múltipla }\end{array}$ \\
\hline
\end{tabular}

GC: grupo controle; Gl: grupo intervenção.

* Tetradeciltioacético: ácido graxo não B-oxidável com potencial efeito de regulação no metabolismo de lipídeos.

\begin{tabular}{|c|c|c|}
\hline Estudo & Resultados CT & Resultados LDL \\
\hline \multicolumn{3}{|l|}{ Experimentais } \\
\hline Barrios e cols. (33) & $\begin{array}{l}\downarrow 11 \% \text { após } 3 \text { meses }(p<0,05) \\
\downarrow 10 \% \text { após } 6 \text { meses }(p<0,05)\end{array}$ & NA \\
\hline Terry e cols. (29) & NS & NA \\
\hline Melroe e cols. (17) & $\downarrow 88 \mathrm{mg} / \mathrm{dL}(p<0,001)$ & NA \\
\hline Lima e cols. (28) & $\downarrow 15,5 \%,(p<0,001)$ & $\downarrow 15,8 \%(p<0,001)$ \\
\hline Moyle e cols. (35) & $\begin{array}{c}\text { GC: } \downarrow \text { } \downarrow 4 \% \text { (não significativo) } \\
\text { Gl: } \downarrow 17 \%(p<0,05), \\
\neq \text { entre grupos: }(p=0,051)\end{array}$ & NS \\
\hline Miller e cols. (25) & NS & NS \\
\hline Mallon e cols. (26) & $\begin{aligned} \mathrm{GC}: & \downarrow \downarrow \downarrow), 34 \mathrm{mmol} / \mathrm{/}, \mathrm{Gl}: \downarrow 0,82 \mathrm{mmol} / \mathrm{l} \\
& \neq \text { entre grupos: }(p=0,04)\end{aligned}$ & NA \\
\hline Ranieri (30) & NS & NS \\
\hline Wohl e cols. (18) & NS & NS \\
\hline Manfredi e cols. (31) & NS & NA \\
\hline $\begin{array}{l}\text { De Truchis e cols. } \\
\text { (32) }\end{array}$ & $\begin{array}{c}\text { Gl: } \downarrow \downarrow 0,4 \%, G C: \uparrow 5,7 \% \\
\text { * entre grupos: }-8,5 \%(p=0,0117)\end{array}$ & NA \\
\hline $\begin{array}{l}\text { Fredriksen e cols. } \\
\text { (34) }\end{array}$ & $\begin{array}{l}\downarrow \text { na fase } 1(p<0,05 \text { vs. baseline }) \\
\downarrow \text { na fase } 2(p<0,05 \text { vs. baseline })\end{array}$ & $\begin{array}{c}\downarrow \text { na fase } 2(p<0,05 \\
\text { vs. baseline })\end{array}$ \\
\hline \multicolumn{3}{|l|}{ Observacionais } \\
\hline $\begin{array}{l}\text { Hadigan e cols. } \\
\text { (19) }\end{array}$ & NS & $\begin{array}{l}\text { 个 consumo de álcool } \\
\text { associado com } \uparrow \text { LDL } \\
\quad(p=0,003)\end{array}$ \\
\hline Gavrila e cols. (20) & NS & NS \\
\hline Shah e cols. (21) & $\begin{array}{l}\text { Correlação positiva com: \% de } \\
\text { proteína da dieta ( } r=0,44 \text {; } \\
p<0,001), \% \text { de proteína animal } \\
(r=0,37 ; p<0,05)\end{array}$ & NA \\
\hline $\begin{array}{l}\text { Floris-Moore e } \\
\text { cols. (22) }\end{array}$ & $\begin{array}{c}\uparrow \text { lipídeos totais na dieta } \\
\text { associados com } \uparrow \text { colesterol total } \\
(p=0,004)\end{array}$ & NS \\
\hline Aghdassi e cols. (36) & NS & NS \\
\hline Wanke e cols. (23) & NS & $\begin{array}{l}\uparrow \text { consumo de fibras } \\
\text { associado com } \downarrow \text { de } \\
\operatorname{LDL}(p=0,035)\end{array}$ \\
\hline Joy e cols. (24) & NS & NA \\
\hline
\end{tabular}

\begin{tabular}{|c|c|}
\hline \multicolumn{2}{|c|}{ Tabela 3. Relação entre dieta e níveis de colesterol HDL } \\
\hline Estudo & Resultados \\
\hline \multicolumn{2}{|l|}{ Experimentais } \\
\hline Terry e cols. (29) & NS \\
\hline Lima e cols. (28) & NS \\
\hline Miller e cols. (25) & NS \\
\hline Mallon e cols. (26) & $\begin{array}{c}\text { GC: PLACEBO } \downarrow \text { } 0,4 \mathrm{mmol} / \mathrm{L} ; \mathrm{Gl}: \downarrow 1 \mathrm{mmol} / \mathrm{L} \\
\quad \neq \text { entre grupos }(p=0,01)\end{array}$ \\
\hline Ranieri (30) & NS \\
\hline Wohl e cols. (18) & NS \\
\hline De Truchis e cols. (32) & NS \\
\hline \multicolumn{2}{|l|}{ Observacionais } \\
\hline Hadigan e cols. (19) & $\begin{array}{l}\text { Associação positiva com consumo de álcool } \\
\qquad(p=0,01)\end{array}$ \\
\hline Gavrila e cols. (20) & NS \\
\hline Shah e cols. (21) & NS \\
\hline Floris-Moore e cols. (22) & Associação positiva com fibras dietéticas $(p<0,03)$ \\
\hline Aghdassi e cols. (36) & NS \\
\hline Wanke e cols. (23) & NS \\
\hline Joy e cols. (24) & NS \\
\hline
\end{tabular}

NS: sem variação/associação significativa; GC: grupo controle; Gl: grupo intervenção; EV: orientações para estilo de vida; AF: orientações para atividade física.

\section{DISCUSSÃO}

As mudanças no estilo de vida, destacando-se dieta e atividade física, são consensualmente indicadas como primeira abordagem no tratamento das dislipidemias, seja em populações não infectadas (37) como em indivíduos vivendo com HIV/AIDS com alterações metabólicas decorrentes da TARV $(8,38,39)$.

A revisão sistemática dos 20 artigos selecionados aponta uma deficiência nos quesitos de qualidade e disponibilidade de estudos que avaliem especificamente os efeitos da dieta e de seus componentes no controle da hipercolesterolemia e hipertrigliceridemia em pacientes soropositivos em terapia antirretroviral. Cinco dos 13 ensaios clínicos estudados são ensaios não controlados 
e, como tal, denotam menor força de evidência. Ademais, observa-se que os estudos, em geral, avaliaram amostras pequenas, variando de 17 a 230 participantes nos estudos experimentais. Em face dessas limitações, observa-se a necessidade de estudos controlados e de tamanho amostral com maior poder de teste para a confirmação dos efeitos previamente observados.

\begin{tabular}{|c|c|}
\hline Estudo & Resultados \\
\hline \multicolumn{2}{|l|}{ Experimentais } \\
\hline Barrios e cols. (33) & $\begin{array}{l}\text { Para pacientes com boa adesão à dieta: } \\
\downarrow 12 \% \text { após } 3 \text { meses }(p<0,05) \downarrow 23 \% \\
\text { após } 6 \text { meses }(p<0,05)\end{array}$ \\
\hline Terry e cols. (29) & NS \\
\hline Melroe e cols. (17) & $\downarrow 439 \mathrm{mg} / \mathrm{dL}(\mathrm{p}<0,001)$ \\
\hline Lima e cols. (28) & $\downarrow 31,0 \%,(p<0,05)$ \\
\hline Moyle e cols.(35) & NS \\
\hline Miller e cols. (25) & $\begin{array}{c}\mathrm{GC}: \uparrow 31 \mathrm{mg} / \mathrm{dL} \\
\mathrm{Gl}: \downarrow 108 \mathrm{mg} / \mathrm{dL} \neq \text { entre grupos: }-139 \mathrm{mg} / \mathrm{dL} \\
(p=0,08)\end{array}$ \\
\hline Mallon e cols. (26) & NS \\
\hline Ranieri (30) & $\downarrow$ TG: $67,3 \mathrm{mg} / \mathrm{dL}(p<0,001)$ \\
\hline Wohl e cols. (18) & $\begin{array}{c}\text { Semana } 4 \text { - GC: } \uparrow 2,8 \% \text {; Gl: } \downarrow 25,1 \% \neq \text { entre } \\
\text { grupos significativa }(p=0,0074) \\
\text { Semana } 16 \text { - GC: } \downarrow 5,7 \% \text {; Gl: } \downarrow 19,5 \% \neq \text { entre } \\
\text { grupos não significativa }(p=0,12)\end{array}$ \\
\hline Manfredi e cols. (31) & $\begin{array}{l}\text { Normalização dos níveis de TG - Gl-2: 25,9\%; } \\
\text { Gl-1: } 34 \% \text {; GC: 8,2\% } \\
\text { * significativa entre GC vs. Gl-1 e Gl-2 ( } p<0,001)\end{array}$ \\
\hline Carter e cols. (27) & $\begin{array}{c}\text { GC: } \downarrow 6 \% \\
\text { Gl: } \downarrow 56,9 \% \text { entre grupos: }-2,32 \mathrm{mmol} / \mathrm{L} \\
(p=0,0487)\end{array}$ \\
\hline De Truchis e cols. (32) & 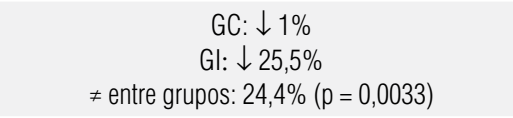 \\
\hline Fredriksen e cols. (34) & $\begin{array}{c}\downarrow \text { na fase } 1(p<0,05 \text { vs. baseline }) ; \downarrow \text { na fase } 2 \\
(p<0,01 \text { vs. baseline, } \\
p<0,05 \text { vs. fase } 1)\end{array}$ \\
\hline \multicolumn{2}{|l|}{ Observacionais } \\
\hline Hadigan e cols. (19) & NS \\
\hline Gavrila e cols. (20) & NS \\
\hline Shah e cols. (21) & $\begin{array}{l}\text { Correlação positiva com: \% de proteína da dieta } \\
\qquad(r=0,40 ; \\
p<0,01) \text {, \% de proteína animal }(r=0,46 ; p<0,01) \text {, } \\
\text { e \% de gordura trans } \\
(r=0,34 ; p<0,05)\end{array}$ \\
\hline Floris-Moore e cols. (22) & NS \\
\hline Aghdassi e cols. (36) & NS \\
\hline Wanke e cols. (23) & NS \\
\hline Joy e cols. (24) & $\begin{array}{l}\uparrow 8,7 \mathrm{mg} / \mathrm{dL} \text { a cada } \uparrow 1 \mathrm{~g} \text { gordura saturada } \\
(p=0,005) \\
\uparrow 3,0 \mathrm{mg} / \mathrm{dL} \text { a cada } \uparrow 1 \mathrm{~g} \text { gorduras totais } \\
(p=0,02)\end{array}$ \\
\hline
\end{tabular}

NS: sem variação/associação significativa; GC: grupo controle; Gl: grupo intervenção; EV: orientações para estilo de vida; AF: orientações para atividade física.

* Tetradeciltioacético: ácido graxo não B-oxidável com potencial efeito de regulação no metabolismo de lipídeos.
O tempo de seguimento é outro ponto a ser considerado, tendo em vista o caráter comportamental da intervenção dietética e a variabilidade entre a mudança no nível do consumo alimentar e o seu impacto nos desfechos metabólicos. Ensaios de longa duração trariam maior consistência para análises do impacto da dieta sobre as alterações lipídicas, porém a viabilidade de tais estudos é questionável tanto pela dificuldade operacional quanto pelas recomendações já estabelecidas de introdução de terapia farmacológica para dislipidemias resistentes após três meses de dietoterapia $(8,37)$. Por outro lado, estudos com maior tempo de seguimento são necessários se a abordagem for a prevenção da ocorrência de tais alterações lipídicas.

Entre os desfechos estudados, as intervenções dietéticas e farmacológicas contemplam resultados de maior magnitude sobre os triglicérides, sobretudo em relação à efetividade da suplementação com $\omega$-3, evidenciandose o papel adjuvante desse ácido graxo poli-insaturado nas dietas para tratamento da hipertrigliceridemia relacionada à TARV. Acerca da hipercolesterolemia, resultados favoráveis da abordagem farmacológica com estatinas foram demonstrados, porém há informação insuficiente sobre o papel específico da dieta. O impacto das intervenções sobre o colesterol HDL é inconsistente, sinalizando mais uma lacuna a ser preenchida por futuros estudos.

As intervenções multicomponentes focadas nas modificações do estilo de vida compõem o escopo das recomendações para a prevenção cardiovascular. Observa-se que análises isoladas dos diferentes componentes de uma intervenção têm sido pouco feitas, e a avaliação global do efeito da intervenção tem sido o foco de análise priorizado. Essa questão limita a síntese de evidências sobre o impacto independentemente de distintos padrões de dieta ou de componentes dietéticos nas dislipidemias em indivíduos em TARV.

A análise dos estudos observacionais incluídos aponta uma ausência de achados conclusivos sobre o papel do consumo alimentar na ocorrência de dislipidemias. A principal limitação desses estudos é o desenho transversal em sua maioria ( 5 entre 7 estudos) que não permite avaliação de causalidade. Também observa-se um possível viés de avaliação do consumo alimentar no estudo de coorte de Joy e cols. (24), que compararam dados obtidos por dois métodos de inquérito alimentar distintos (diário alimentar e recordatório de 24 horas).

No sentido de contribuir com a formação de diretrizes para a prevenção e controle das dislipidemias secun- 
dárias à terapia antirretroviral em pessoas infectadas pelo HIV, os achados desta revisão indicam efeito positivo da suplementação com $\omega$-3 sobre os triglicérides séricos; com menor segurança, papel protetor do consumo de fibras, e risco de que o consumo de lipídeos e proteínas de origem animal aumente a concentração de LDL-c. Por outro lado, observa-se insuficiente evidência científica acerca de um modelo de intervenção nutricional eficaz. Identificaram-se quantidade escassa e qualidade metodológica inadequada de pesquisas delineadas para avalizar o efeito da dieta e, consequentemente, a latente necessidade de mais estudos nesta área.

Finalmente, esta revisão chama atenção para a necessidade do direcionamento de pesquisas: (i) a comprovação científica com determinação de dose-resposta e magnitude de efeitos colaterais da suplementação com ácidos graxos $\omega-3$ no tratamento da hipertrigliceridemia; (ii) elucidação do efeito independente de diferentes intervenções dietéticas no controle das dislipidemias; (iii) esclarecimentos sobre o papel de nutrientes específicos como fibras dietéticas, proteínas, gorduras totais, saturadas, insaturadas e trans como fatores de risco ou proteção para as alterações lipídicas relacionadas à TARV.

Agradecimentos: Conselho Nacional de Desenvolvimento Científico e Tecnológico $(\mathrm{CNPq})$ pela bolsa de iniciação científica do Programa Institucional de Bolsas de Iniciação Científica (PIBIC) da autora Kelly Virecoulon Giudici. Por se tratar de uma revisão de literatura, o estudo não foi submetido ao Comitê de Ética em Pesquisa da instituição.

Declaração: os autores declaram não haver conflito de interesse científico neste artigo.

\section{REFERÊNCIAS}

1. Sterne JAC, Hernán MA, Ledergerber B, Tilling $K$, Weber $R$, Sendi $P$, et al. Long-term effectiveness of potent antiretroviral therapy in preventing AIDS and death: a prospective cohort study. Lancet. 2005;366(9483):378-84.

2. Dourado I, Veras MASM, Barreira D, Brito AM. AIDS epidemic trends after the introduction of antiretroviral therapy in Brazil. Rev Saude Publica. 2006;40(Suppl):9-17.

3. Grinspoon S, Carr A. Cardiovascular risk and body fat abnormalities in HIV infected adults. N Engl J Med. 2005;352(1):48-62.

4. Valente AMM, Reis AF, Machado DM, Succi RCM, Chacra AR. Alterações metabólicas da síndrome lipodistrófica do HIV. Arq Bras Endocrinol Metab. 2005;49(6):871-81.

5. Friis-Møller N, Sabin CA, Weber R, d'Arminio Monforte A, El-Sadr WM, Reiss $P$, et al. Combination antiretroviral therapy and the risk of myocardial infarction. N Engl J Med. 2003:349(21):1993-2003.

6. Savès $M$, Chêne $G$, Ducimetière $P$, Leport $C$, Le Moal G, Amouyel $P$, et al. Risk factors for coronary heart disease in patients treated for human immunodeficiency virus infection compared with the general population. Clin Infect Dis. 2003;37(2):292-8.
7. Boccara F, Cohen A, Di Angelantonio E, Meuleman C, Ederhy S, Dufaitre G, et al. Coronary artery bypass graft in HIV-infected patients: a multicenter case control study. Curr HIV Res. 2008;6(1):59-64.

8. Carosi G, Quiros-Roldan E, Torti C, Antinori A, Bevilacqua M, Bonadonna $\mathrm{RC}$, et al. First Italian consensus statement on diagnosis, prevention and treatment of cardiovascular complications in HIV-infected patients in the HAART era (2006). Infection. 2007;35(3):134-42.

9. Phillips AN, Carr A, Neuhaus J, Visnegarwala F, Prineas R, Burman WJ, et al. Interruption of antiretroviral therapy and risk of cardiovascular disease in persons with HIV-1 infection: exploratory analyses from the SMART trial. Antivir Ther. 2008;13(2):177-87.

10. Hadigan C. Dietary habits and their association with metabolic abnormalities in human immunodeficiency virus - related lipodystrophy. Clin Infect Dis. 2003;37(Suppl 2):S101-4.

11. Leyes $\mathrm{P}$, Martínez E, Forga MT. Use of diet, nutritional supplements and exercise in HIV-infected patients receiving combination antiretroviral therapies: a systematic review. Antivir Ther. 2008;13(2):149-59.

12. Jones SP, Doran DA, Leatt PB, Maher B, Pirmohamed M. Shortterm exercise training improves body composition and hyperlipidaemia in HIV-positive individuals with lipodystrophy. AIDS. 2001;15(15):2049-51.

13. Mutimura E, Crowther NJ, Cade TW, Yarasheski KE, Stewart A. Exercise training reduces central adiposity and improves metabolic indices in HAART-treated HIV-positive subjects in Rwanda: a randomized controlled trial. AIDS Res Hum Retroviruses. 2008;24(1):15-23.

14. Van Horn L, McCoin M, Kris-Etherton PM, Burke F, Carson JA, Champagne $\mathrm{CM}$, et al. The evidence for dietary prevention and treatment of cardiovascular disease. J Am Diet Assoc. 2008;108(2):287-331.

15. World Health Organization (WHO). Diet, nutrition and the prevention of chronic diseases. Report of a joint WHO/FAO Consultation. Geneva:WHO; 2003. Technical Report Series; n. 916.

16. Stein JH. Managing cardiovascular risk in patients with HIV infection. J Acquir Immune Defic Syndr. 2005;38(2):115-23.

17. Melroe N, Kopaczewski J, Henry K, Huebsch J. Intervention for hyperlipidemia associated with protease inhibitors. J Assoc Nurses AIDS Care. 1999;10(4):55-69.

18. Wohl D, Tien H, Busby M, Cunningham C, Macintosh B, Napravnik $S$, et al. Randomized study of the safety and efficacy of fish oil (omega-3 fatty acid) supplementation with dietary and exercise counseling for the treatment of antiretroviral therapy-associated hypertriglyceridemia. Clin Infect Dis. 2005;41(10):1498-504.

19. Hadigan C, Jeste S, Anderson E, Tsay R, Cyr H, Grinspoon S. Modifiable dietary habits and their relation to metabolic abnormalities in men and women with human immunodeficiency virus infection and fat redistribution. Clin Infect Dis. 2001;33(5):710-7.

20. Gavrila A, Tsiodras S, Doweiko J, Nagy G, Brodovicz K, Hsu W, et al. Exercise and vitamin $\mathrm{E}$ intake are independently associated with metabolic abnormalities in human immunodeficiency virus-positive subjects: a cross-sectional study. Clin Infect Dis. 2003;36(12):1593-601.

21. Shah M, Tierney $K$, Adams-Huet B, Boonyavarakul A, Jacob $K$, Quittner $\mathrm{C}$, et al. The role of diet, exercise and smoking in dyslipidaemia in HIV-infected patients with lipodystrophy. HIV Med. 2005;6(4):291-8.

22. Floris-Moore M, Howard A, LoY, Arnsten J, Santoro N, Schoenbaum E. Increased serum lipids are associated with higher CD4 lymphocyte count in HIV-infected women. HIV Med. 2006;7(7):421-30.

23. Wanke C, Gerrior J, Hendricks K, McNamara J, Schaefer E. Alterations in lipid profiles in HIV-infected patients treated with protease inhibitor therapy are not influenced by diet. Nutr Clin Pract. 2005;20(6):668-73. 
24. Joy T, Keogh H, Hadigan C, Lee H, Dolan S, Fitch K, et al. Dietary fat intake and relationship to serum lipid levels in HIV-infected patients with metabolic abnormalities in the HAART era. AIDS. 2007;21(12):1591-600.

25. Miller J, Brown D, Amin J, Kent-Hughes J, Law M, Kaldor J, et al. A randomized, double-blind study of gemfibrozil for the treatment of protease inhibitor-associated hypertriglyceridaemia. AIDS. 2002;16(16):2195-200.

26. Mallon P, Miller J, Kovacic J, Kent-Hughes J, Norris R, Samaras $\mathrm{K}$, et al. Effect of pravastatin on body composition and markers of cardiovascular disease in HIV-infected men - a randomized, placebo-controlled study. AIDS. 2006;20(7):1003-10.

27. Carter R, Wiener J, Abrams E, Farley J, Nesheim S, Palumbo P, et al. Dyslipidemia among perinatally HIV-infected children enrolled in the PACTS-HOPE cohort, 1999-2004: a longitudinal analysis. J Acquir Immune Defic Syndr. 2006;41(4):453-60.

28. Lima E, Gualandro D, Yu P, Giuliano I, Marques A, Calderaro D, et al. Cardiovascular prevention in HIV patients: results from a successful intervention program. Atherosclerosis. 2008 Aug. [Epub ahead of print].

29. Terry L, Sprinz E, Stein R, Medeiros N, Oliveira J, Ribeiro J. Exercise training in HIV-1-infected individuals with dyslipidemia and lipodystrophy. Med Sci Sports Exerc. 2006;38(3):411-7.

30. Ranieri R. Effect of omega-3 fatty acids on the lipid structure of HIV-positive patients on antiretroviral therapy: personal observation of 20 subjects. Infez Med. 2007;15(4):237-41.

31. Manfredi R, Calza L, Chiodo F. Polyunsaturated ethyl esters of $n-3$ fatty acids in HIV-infected patients with moderate hypertriglyceridemia: comparison with dietary and lifestyle changes, and fibrate therapy. J Acquir Immune Defic Syndr. 2004;36(3):878-80.

32. De Truchis $P$, Kirstetter M, Perier A, Meunier C, Zucman D, Force $G$, et al. Reduction in triglyceride level with $\mathrm{N}-3$ polyunsatura- ted fatty acids in HIV-infected patients taking potent antiretroviral therapy: a randomized prospective study. J Acquir Immune Defic Syndr. 2007;44(3):278-85.

33. Barrios A, Blanco F, Garcia-Benayas T, Gomez-Viera J, de la Cruz J, Soriano $V$, et al. Effect of dietary intervention on highly active antiretroviral therapy-related dyslipemia. AIDS. 2002;16(15):2079-81.

34. Fredriksen J, Ueland T, Dyroy E, Halvorsen B, Melby K, Melbye L, et al. Lipid-lowering and anti-inflammatory effects of tetradecylthioacetic acid in HIV-infected patients on highly active antiretroviral therapy. Eur J Clin Invest. 2004;34(10):709-15.

35. Moyle G, Lloyd M, Reynolds B, Baldwin C, Mandalia S, Gazzard B. Dietary advice with or without pravastatin for the management of hypercholesterolaemia associated with protease inhibitor therapy. AIDS. 2001;15(12):1503-8.

36. Aghdassi E, Salit I, Fung L, Sreetharan L, Walmsley S, Allard J. Is chromium an important element in HIV-positive patients with metabolic abnormalities? An hypothesis generating pilot study. J Am Coll Nutr. 2006;25(1):56-63.

37. National Cholesterol Education Program (NCEP). Executive Summary of the Third Report of the National Cholesterol Education Program (NCEP) Expert Panel on Detection, Evaluation, andTreatment of High Blood Cholesterol in Adults (Adult Treatment Panel III). JAMA. 2001;285(19):2486-97.

38. American Dietetic Association (ADA). Position of the American Dietetic Association and Dietitians of Canada: Nutrition intervention in the care of persons with human immunodeficiency virus infection. J Am Diet Assoc. 2004;104:1425-41.

39. Dubé MP, Stein JH, Aberg JA, Fichtenbaum CJ, Gerber JB, Tashima $\mathrm{KT}$, et al. Guidelines for the evaluation and management of dyslipidaemia in human immunodeficiency virus (HIV) -infected adults receiving antiretroviral therapy: recommendations of the HIV Medicine Association of the Infectious Disease Society of America and the Adult Aids Clinical Trials Group. Clin Inf Dis. 2003;37:613-27. 